\title{
Ab initio Monte Carlo simulations for finite-temperature properties: Application to lithium clusters and bulk liquid lithium
}

\author{
Sanwu Wang, ${ }^{1,2, \text { * }}$ Steven J. Mitchell, ${ }^{2,3}$ and Per Arne Rikvold ${ }^{2}$ \\ ${ }^{1}$ Department of Physics and Astronomy, Vanderbilt University, Nashville, Tennessee 37235 \\ ${ }^{2}$ School of Computational Science and Information Technology, \\ and Center for Materials Research and Technology, and Department of Physics, \\ Florida State University, Tallahassee, Florida 32306-4120 \\ ${ }^{3}$ Laboratory of Inorganic Chemistry and Catalysis, \\ Eindhoven University of Technology, 5600 MB Eindhoven, The Netherlands
}

\begin{abstract}
$A b$ initio Monte Carlo simulations have been performed to determine the equilibrium properties of liquid lithium and lithium clusters at different temperatures. First-principles density-functional methods were employed to calculate the potential-energy change for each proposed change of configuration, which was then accepted or rejected according to the Metropolis Monte Carlo scheme. The resulting structural properties are compared to data from experimental measurements and ab initio molecular dynamics simulations. It is shown that accurate structural information can be obtained with ab initio Monte Carlo simulations at computational costs comparable to ab initio molecular dynamics methods. We demonstrate that ab initio Monte Carlo simulations for the properties of fairly large condensed-matter systems at nonzero temperatures are feasible.
\end{abstract}

Keywords: Ab initio calculations; Monte Carlo simulations; Density-functional theory; Lithium; Clusters; Liquid; Pair correction functions

\section{INTRODUCTION}

Molecular dynamics (MD) and Monte Carlo (MC) simulations have been the major techniques for calculating finite-temperature properties of condensed-matter systems. In particular, ab initio MD simulations, which combine classical molecular dynamics with firstprinciples quantum theory, determine the atomic and electronic structures of a system simultaneously and accurately, as well as the temperature-dependent properties and time evolution of the system.1.2 $A b$ initio MD methods have been widely used to provide reliable and accurate information for various systems since Car and Parrinello first introduced their work in $1985^{1}$

On the other hand, ab initio Monte Carlo simulations, which employ first-principles quantum theory to calculate the potential energy of a system at each classical MC step, have so far been used only to a very limited extent. The majority of MC simulations for condensedmatter systems have been performed with empirical or semiempirical atom-atom interaction potentials, which are fitted to available experimental data or ab initio calculations. While the atom-atom potentials may work well in some cases, they do not provide reliable results in other instances. Since the quantum many-body effects and the detailed electronic structures cannot be included in empirical or semi-empirical potentials, firstprinciples theory would obviously be desirable for providing reliable energetics. The major concern with $a b$ initio Monte Carlo simulations is the high computational demand. In particular, Monte Carlo simulations with empirical potentials often generate millions of sampling configurations. It is a formidable task to determine the potential energies of such a huge number of configurations with $a b$ initio total-energy calculations. In recent years, however, several groups have performed $a b i n i$ tio MC simulations for very small clusters, and they have tried to reduce the number of sampling configura-

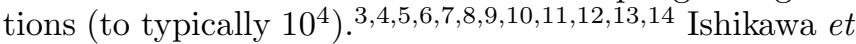
$a l^{3.12 .14}$ applied $a b$ initio calculations to the Monte Carlo simulated annealing algorithm 15 . They performed simulations for the $\mathrm{Li}_{6}$ cluster, small hydrogenated lithium clusters $\left(\mathrm{Li}_{5} \mathrm{H}, \mathrm{Li}_{4} \mathrm{H}_{2}\right.$, and $\left.\mathrm{Li}_{7} \mathrm{H}\right)$, and $\mathrm{HCl}\left(\mathrm{H}_{2} \mathrm{O}\right)_{n}(n=$ $3,4)$ clusters 3.12 .14 Other groups ${ }^{4.5 .7 .10}$ used the $a b$ initio molecular orbital (MO) method in classical MC simulations to study the interactions of ions and molecules with small water clusters. Structural and thermal properties of water dimers 11 and lithium clusters $\left(\mathrm{Li}_{4}, \mathrm{Li}_{5}^{+}\right.$, and $\left.\mathrm{Li}_{8}\right)^{8.9 .13}$ were also investigated with ab initio MC simulations.

We emphasize that all the previous ab initio MC simulations were limited to very small clusters. Since experimental data for the structures of the clusters at nonzero temperatures were not available, direct comparisons of the above simulations with experiments were not possible. Therefore, the accuracy and efficiency of $a b$ initio $\mathrm{MC}$ simulations remain to be determined. Furthermore, the feasibility of the extension of $a b$ initio MC simulations to bulk systems, which require models containing many more atoms than the small clusters, has yet to be tested. As a first application of $a b$ initio MC simulations to bulk systems, we have chosen the liquid phase of bulk lithium. We have also investigated the structural properties of a cluster containing 16 lithium atoms at different temperatures. The simulated results for the lithium cluster are physically reasonable, and the structure of liquid lithium obtained from our ab initio MC simulations is in good agreement with the available experimental measurements. We also demonstrate that the computational demands of simulating liquid lithium with our $a b$ initio 
$\mathrm{MC}$ algorithms are comparable to those of ab initio $\mathrm{MD}$ simulations.

The remainder of this paper is organized as follows. Section II describes our $a b$ initio MC algorithm. Section III describes the simulation results for a 16-atom lithium cluster (Sec. III A) and for liquid bulk lithium (Sec. III $\mathrm{B})$, with a discussion of general aspects and possible improvements of our ab initio MC algorithm in Sec. III C. Sec. IV contains our conclusion.

\section{AB INITIO MONTE CARLO ALGORITHM}

To simulate the cluster of 16 lithium atoms, the atoms were put in a periodically repeated cubic supercell. The size of the supercell $\left(10 \times 10 \times 10 \AA^{3}\right)$ was much larger than the concentrated cluster (see Fig. 1.). Bulk lithium was represented by a cubic box (supercell) with periodic boundary conditions containing $54 \mathrm{Li}$ atoms. The size of the supercell $\left(10.698 \times 10.698 \times 10.698 \AA^{3}\right.$ and $10.874 \times 10.874 \times 10.874 \AA^{3}$ for simulations at $T=523 \mathrm{~K}$ and $T=725 \mathrm{~K}$, respectively) was determined to match the experimental density of liquid lithium at the given temperature. 16

The simulations were performed in canonical ensembles. The standard Metropolis algorithm, 17.18 which provides an efficient approach for simulating the equilibrium properties of an atomic system at a given temperature, was used in our ab initio MC simulations. Starting from a pre-selected initial configuration, the simulations repeated the following steps. (1) Given the current configuration $I$ of the system, a new configuration $J$ is generated by making a small random displacement of one randomly chosen particle (suitable for small systems) or random displacements of all the particles simultaneously (more efficient for systems containing a large number of particles). (2) Once the total energies $\left(E_{I}\right.$ and $\left.E_{J}\right)$ of these two configurations are calculated, the acceptance probability of the new configuration $J$ is then determined as

$$
P(J \mid I)=\min \left[1, \exp \left(\frac{-\left(E_{J}-E_{I}\right)}{k_{B} T}\right)\right],
$$

where $T$ is the temperature and $k_{B}$ is Boltzmann's constant. (3) If the configuration $J$ is accepted, it serves as the current configuration of the next MC step, and $E_{I}$ is set equal to $E_{J}$. If the configuration $J$ is not accepted, the configuration $I$ and its energy $E_{I}$ are retained and used to start the next step. In this way, the system will eventually reach equilibrium and evolve toward a Boltzmann distribution. ${ }^{17,18}$ The resulting sequence of sampling configurations is then used to obtain the equilibrium properties of the system.

To calculate the total energy at each MC step, we employed an $a b$ initio pseudopotential density-functionaltheory (DFT) method with a plane-wave basis set. All the $a b$ initio total-energy calculations were carried out using the Vienna ab-initio simulation package
(VASP) $19,20,21$ The electronic exchange-correlation effects were treated with the generalized gradient-corrected functionals given by Perdew and Wang ${ }^{22.23}$ We adopted the Vanderbilt ultrasoft pseudopotential to replace the core electrons ${ }^{24.25}$ and used the conjugate-gradient technique for performing electronic relaxations ${ }^{2}$. The totalenergy calculations were conducted with $4 \mathrm{k}$-points in the three-dimensional Brillouin zone with a plane-wave energy cutoff of $120 \mathrm{eV}$. The convergence of the total energy differences between different configurations was checked for selected configurations with higher cutoff energies (up to $200 \mathrm{eV}$ ) and more k-points (up to $16 \mathbf{k}$ points), and the convergence was found to be within a few meV. Because we used a pseudopotential approach, the total-energy calculations for the configurations with too small distances between atoms, which result in corecore overlap, would give wrong total energies. We therefore defined a hard-core distance of $2.0 \AA$ between any two Li atoms. Trial configurations with any interatomic distance smaller than the cutoff distance were always rejected in the MC simulations. This is physically reasonable because such configurations would have very high potential energies due to the strong core-core repulsion.

\section{RESULTS AND DISCUSSION}

\section{A. Small cluster}

Starting from the configuration with the atoms at ordered bcc positions (shown in Fig. 1), we first performed our ab initio MC simulations for the cluster of 16 lithium atoms at temperatures of $1000 \mathrm{~K}$ and $5000 \mathrm{~K}$. The MC moves in the simulations for the cluster consisted of random displacement of one randomly selected lithium atom at each MC step, with the maximum displacement adjusted to give an overall acceptance rate of about $50 \%$. The chosen atom was displaced from its old position with equal probability to any position inside a sphere surrounding the old position, with the radius of the sphere being the maximum displacement. We obtained maximum displacements of $0.75 \AA$ and $1.2 \AA$ for the simulations at the temperatures of $1000 \mathrm{~K}$ and $5000 \mathrm{~K}$, respectively. About $10000 \mathrm{MC}$ steps were needed to reach the equilibrium state. Averages for calculating the structural properties were taken over the next 10000 sampling configurations after equilibration. It took approximately 1 minute of CPU time to obtain the total energy of each configuration with a single $375 \mathrm{MHz}$ processor on an IBM SP3 supercomputer.

The pair correlation function $g(r)$ can be defined $\mathrm{as}^{26}$

$$
g(r)=\frac{2 V}{N(N-1)} \frac{1}{4 \pi r^{2}}\left\langle\sum_{i} \sum_{j<i} \delta\left(r-r_{i j}\right)\right\rangle
$$

where $V$ is the system volume, $N$ is the number of particles, $r_{i j}$ is the distance between a pair of particles $i$ and $j$, and $\langle\cdot\rangle$ signifies averaging over the sequence of simulated 


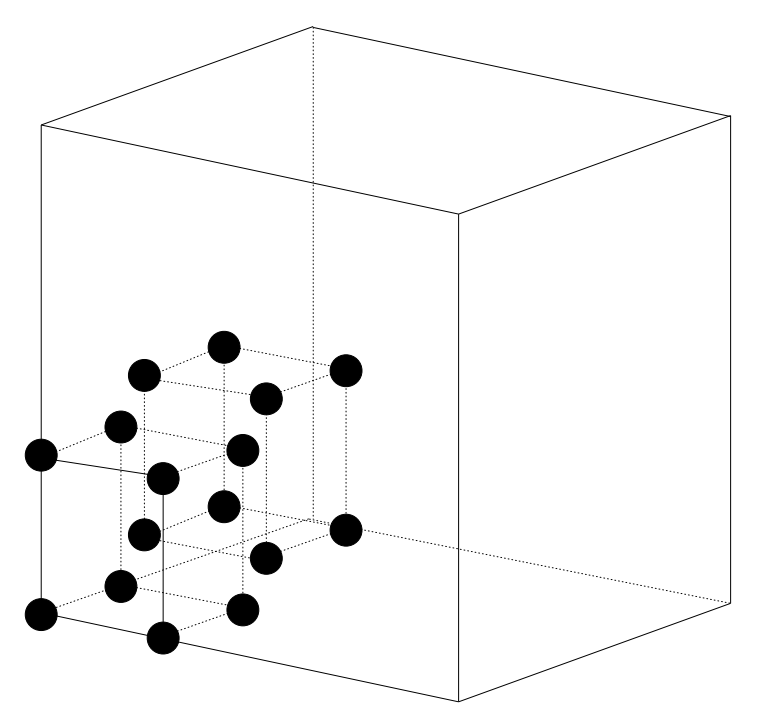

FIG. 1: The initial configuration of the cluster of 16 lithium atoms for the $a b$ initio MC simulations. The distance between two nearest-neighbor atoms is $2.96 \AA$, the same as that determined by our $a b$ initio total-energy calculations for the crystalline bulk Li. The size of the supercell is $10 \times 10 \times 10$ $\AA^{3}$.

configurations. The pair correlation function is proportional to the probability of finding a pair of particles a distance $r$ apart, normalized by the square of the particle density such that it approaches unity in the limit of a uniform random distribution at the same density. It hence provides a measure of the local spatial ordering of the system. As described in Ref. 26, we determined $g(r)$ by dividing the range of $r([2 \AA, 10 \AA])$ into 400 intervals of length $0.02 \AA(\Delta r)$, calculating every $r_{i j}$ for a given configuration, and recording in narrow bins $[r, r+\Delta r)$ the frequency of occurrence of the different particle separations. In this way a histogram was built up and finally normalized at the end of the scan over the configurations.

Figure 2 shows the pair correlation functions for the cluster at temperatures of $1000 \mathrm{~K}$ and $5000 \mathrm{~K}$, respectively. It can be observed that there are two peaks in the pair correlation function at $T=1000 \mathrm{~K}$. This indicates the existence of two atomic shells for the structure, and therefore the system locally has a liquid-like structure. At a temperature of $5000 \mathrm{~K}$, however, there is no obvious second peak in the pair correlation function, and thus the system has a gas-like structure. Given that the boiling point of bulk $\mathrm{Li}$ is about $1600 \mathrm{~K}$, the results from our simulations for the cluster of lithium atoms are physically reasonable. Note that the pair correlation function $g(r)$ at $T=5000 \mathrm{~K}$ does not approach zero when $r$ is close to $2.0 \AA$, indicating that the configurations with very small separations (smaller than $2.0 \AA$ ) between atoms would contribute to the ensemble averages. This reflects the gas-like structure of the system at $T=5000 \mathrm{~K}$ because the probability of occurrence of configurations with very small separations between particles is expected to be higher for a gas-like structure than for a liquid-like

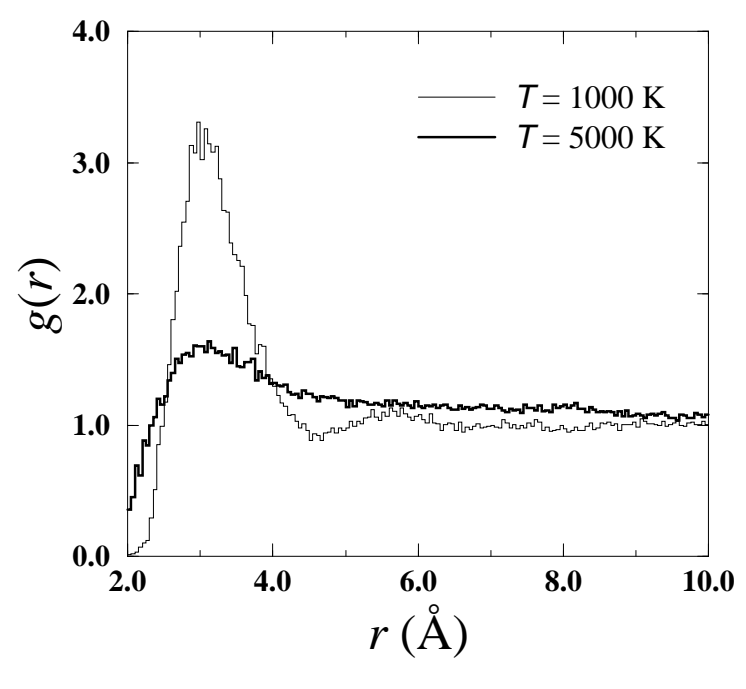

FIG. 2: Equilibrium pair correlation functions $g(r)$ for a cluster of 16 lithium atoms at temperatures of $1000 \mathrm{~K}$ and $5000 \mathrm{~K}$, obtained from our ab initio Monte Carlo simulations.

structure.

\section{B. Bulk system}

Following the simulations for the lithium cluster, we carried out ab initio MC simulations for the liquid phase of bulk lithium. In contrast to the simulations for the lithium cluster, the Monte Carlo moves in the simulations for liquid lithium consisted of random displacements of all the atoms in the supercell simultaneously. The other aspects of the MC moves were the same as in the simulations for the lithium cluster. In order to achieve an overall acceptance rate of roughly $50 \%$, we found that the maximum displacements at temperatures of $523 \mathrm{~K}$ and $725 \mathrm{~K}$ were $0.05 \AA$ and $0.06 \AA$, respectively. We started our simulations at $T=725 \mathrm{~K}$ from an initial configuration with all the Li atoms at their bulk bcc positions. Roughly 8000 MC steps were required to reach equilibrium. Since the initial configuration was a perfect crystalline structure, far away from the equilibrium structure of the liquid phase of bulk lithium, it required relatively long $\mathrm{MC}$ runs to reach equilibrium. In contrast, the initial configuration for the simulations at $T=523 \mathrm{~K}$ was chosen as one of the equilibrium sampling configurations obtained in the simulations at $T=725 \mathrm{~K}$. A reduced number of MC steps (5000 MC steps) was then required for the equilibration period. After equilibration at either temperature, we generated 5000 sampling configurations for averages.

Our simulations were carried out in parallel on four $375-\mathrm{MHz}$ processors of an IBM SP3 supercomputer. The total-energy calculation at each MC step required approximately 150 seconds of CPU time. On average, the electronic relaxation converged within seven self- 


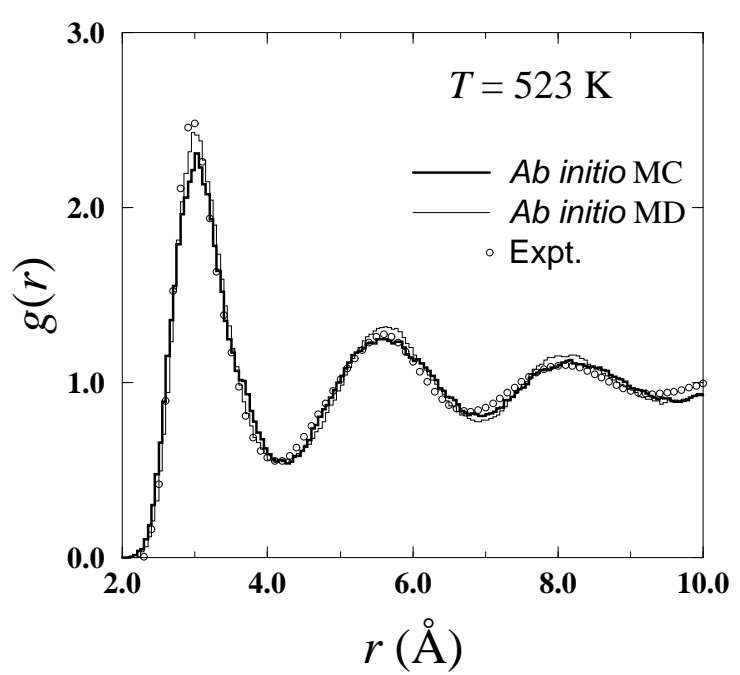

FIG. 3: Equilibrium pair pair correlation function $g(r)$ for liquid lithium at a temperature of $523 \mathrm{~K}$, calculated from $a b$ initio Monte Carlo and ab initio molecular dynamics simulations (histograms). Also shown are experimental data measured by X-ray diffraction (circles) ${ }^{28}$

consistency steps. The software (VASP) that we used for the total-energy calculations contains computations for the forces after the electronic relaxation converges. Such computations are not needed in ab initio MC simulations. Should VASP be modified to exclude the force calculations, the computational costs would therefore be decreased by approximatively $10 \%-15 \%$.

For comparison, we also performed ab initio MD simulations ${ }^{19.20 .21}$ for liquid lithium. Canonical ensembles were generated with the algorithm of Nosé, 27 and the time step was set to $1 \mathrm{fs}$. The initial configuration for the $a b$ initio MD simulations at both $T=725 \mathrm{~K}$ and $T=$ $523 \mathrm{~K}$ was the same perfect bcc structure as that for the $a b$ initio $\mathrm{MC}$ simulations at $T=725 \mathrm{~K}$. The equilibration periods extended over 5000 and 3000 time steps at $T=$ $523 \mathrm{~K}$ and $T=725 \mathrm{~K}$, respectively. Ensemble averages were calculated from the sampling configurations of the next 5000 steps. Each step required about 400 seconds of CPU time on an IBM SP3 supercomputer (in parallel on 4 processors), and the corresponding wall-clock time was roughly 100 seconds.

The pair correlation functions for liquid lithium at temperatures of $523 \mathrm{~K}$ and $725 \mathrm{~K}$, obtained from our $a b$ initio $\mathrm{MC}$ and ab initio MD simulations are shown in Fig. 3 and Fig. 4, respectively. For comparison, the experimental data for liquid lithium at $T=523 \mathrm{~K} \mathrm{ob-}$ tained by X-ray diffraction 28 are also shown in Fig. 3. We observe that the agreement between ab initio MC, $a b$ initio $\mathrm{MD}$, and experiment is very good. In particular, the three peaks determined from our ab initio MC simulations are essentially located at the experimental positions. The intensities of the peaks obtained from the ab initio MC simulations are also basically in agree-

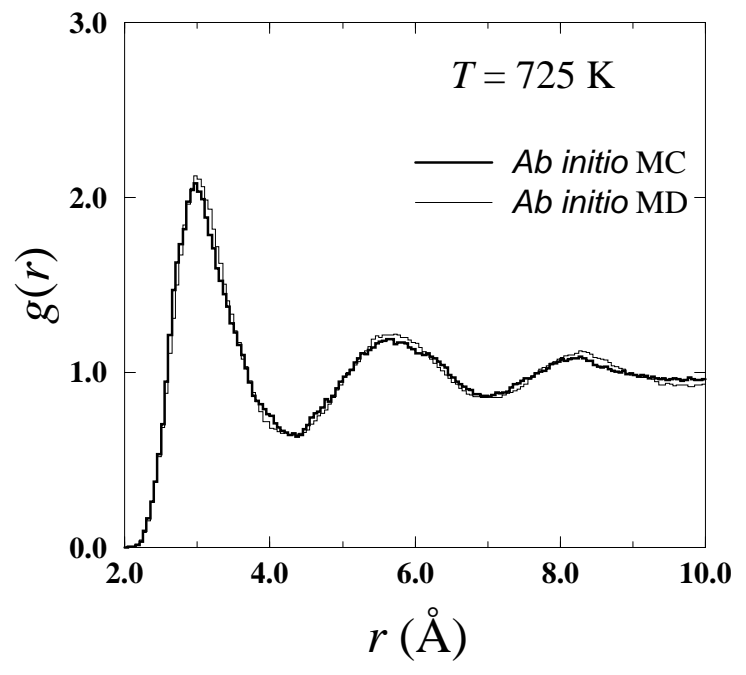

FIG. 4: Equilibrium pair correlation function $g(r)$ for the liquid phase of lithium at a temperature of $725 \mathrm{~K}$, obtained from $a b$ initio MC and $a b$ initio MD simulations.

ment with those from both the $a b$ initio MD simulations and the X-ray data. The small differences of the intensities of the peaks between the theoretical results and the experimental data may be due to the small size of our supercells. The experimentally observed temperature effects, ${ }^{29}$ that is, broadening of the peaks and slight shifts to higher positions of the second and third peaks at the higher temperature are also well reproduced by our $a b$ initio MC simulations (see Fig. 3 and Fig. 4).

\section{General discussion}

Finally, we discuss some general aspects and possible improvements of our ab initio MC algorithm. The computational costs of ab initio MC simulations are dominated by the $a b$ initio total-energy calculations. Since efficient iterative schemes for ab initio total-energy calculations with a plane-wave basis set have achieved order $N^{2}$ ( $N$ is the number of the atoms in the supercell) scal-

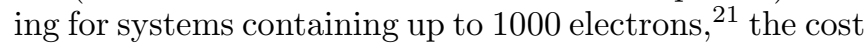
of simulations with our $a b$ initio MC algorithm would increase essentially as the square of the number of atoms.

While the computational costs are comparable, our $a b$ initio MC algorithm is not any faster than ab initio MD simulations. In particular, a larger number of steps are required to reach equilibrium in our ab initio MC simulations for bulk lithium than in the corresponding $a b$ initio MD simulations. For the lithium systems that we have investigated, our ab initio MC algorithm produces the required sampling configurations at a speed similar to the $a b$ initio MD simulations. In this respect, our $a b$ initio MC simulations are as efficient as ab initio MD simulations.

In some cases, like simulations for strongly covalent 
systems and situations involving volume changes, however, our ab initio MC algorithm may not be efficient due to the low acceptance rate of the traditional MC moves. In such situations, therefore, our ab initio MC algorithm needs to be improved. A cluster MC move scheme proposed by Lee and Swendsen ${ }^{30}$ may be used for this purpose. We have tested the cluster MC move scheme for the bulk lithium systems that we investigated with traditional MC moves as described in Sec. III. We found that the use of cluster MC moves in ab initio MC simulations provided almost the same performance as the traditional MC moves, indicating that both kinds of MC moves might work equally well for metallic systems. It is also possible that the relative performance of different kinds of MC moves somewhat depend on the number of particles included in the supercell. It would be interesting to see if the cluster MC move scheme would be more efficient than the traditional MC approach in simulations for systems containing large numbers (for example, hundreds) of particles. In the cases where the boundary conditions or/and the volume of the simulated cells change, which result in a low acceptance rate with the traditional MC moves, the cluster MC move scheme has been shown to increase the acceptance rate significantly ${ }^{30}$ We also expect that the cluster MC move scheme would be more efficient than the traditional MC move method in simulations for systems containing strong covalent bonds. Another approach, a hybrid of MD and MC simulation in which trial MC moves are generated with classical MD methods, may also enhance the efficiency of ab initio MC simulations 31 Other techniques aiming at sampling important configurations in the configuration space include the J-walking procedure proposed by Frantz et al ${ }^{9,13,32}$ and a big-move method suggested by Akhmatskaya et al. $\underline{\underline{6}}$.

\section{CONCLUSION}

In conclusion, we have shown that the use of $a b$ initio calculations in classical Monte Carlo simulations provides structures of liquid lithium in agreement with experimental measurements and ab initio MD simulations. Our work also demonstrates that $a b$ initio MC simulations for condensed-matter systems with up to several tens of atoms (and possibly with more than a hundred atoms) are feasible and may provide an alternative way to investigate finite-temperature properties of such systems.

\section{Acknowledgments}

We thank G. Brown, Y.Z. Cao, Z. Liu, M.A. Novotny, and H.K. Lee for helpful discussions. This work was supported by the National Science Foundation under grants DMR-9981815, DMR-0111841, and DMR-0240078, and by Florida State University through the Center for Materials Research and Technology and the School of Computational Science and Technology. The calculations were performed on IBM SP3 computers at Florida State University and the National Energy Research Scientific Computing Center, which is supported by the U.S. Department of Energy.
* Corresponding author. Tel.: +1 615 3432119; fax: +1 615 3437697. E-mail address: sanwu.wang@vanderbilt.edu (Sanwu Wang)

1 R. Car and M. Parrinello, Phys. Rev. Lett. 55, 2471 (1985).

2 M. C. Payne, M. P. Teter, D. C. Allan, T. A. Arias, and J. D. Joannopoulos, Rev. Mod. Phys. 64, 1045 (1992).

${ }^{3}$ V. Keshari and Y. Ishikawa, Chem. Phys. Lett. 218, 406 (1994).

4 T. N. Truong and E. V. Stefanovich, Chem. Phys. Lett. 256, 348 (1996).

5 T. Asada and S. Iwata, Chem. Phys. Lett. 260, 1 (1996).

${ }^{6}$ E. V. Akhmatskaya, M. D. Cooper, N. A. Burton, A. J. Masters, and I. Hillier, Chem. Phys. Lett. 267, 105 (1997).

7 D. A. Estrin, J. Kohanoff, D. H. Laria, and R. O. Weht, Chem. Phys. Lett. 280, 280 (1997).

8 R. O. Weht, J. Kohanoff, D. A. Estrin, and C. Chakravarty, J. Chem. Phys. 108, 8848 (1998).

9 J. Jellinek, S. Srinivas, and P. Fantucci, Chem. Phys. Lett. 288, 705 (1998).

10 H. Watanabe and T. Asada, Chem. Phys. 237, 81 (1998).

11 P. Bandyopadhyay, S. Ten-No, and S. Iwata, Mol. Phys. 96, 349 (1999).

${ }^{12}$ D. E. Bacelo, R. C. Binning, Jr., and Y. Ishikawa, J. Phys. Chem. A 103, 4631 (1999).
13 S. Srinivas and J. Jellinek, Phys. Stat. Sol. (b) 217, 311 (2000).

14 Y. Ishikawa, Y. Sugita, T. Nishikawa, and Y. Okamoto, Chem. Phys. Lett. 333, 199 (2001).

15 S. Kirkpatrick, C. D. Gelatt, Jr., and M. P. Vecchi, Science 220, 4598 (1983).

16 R. W. Ohse (ed.), Handbook of Thermodynamic and Transport Properties of Alkali Metals (The Alden Press, Oxford, UK, 1985).

17 N. Metropolis, A. Rosenbluth, M. Rosenbluth, A. Teller, and E. Teller, J. Chem. Phys. 21, 1087 (1953).

18 D. P. Landau and K. Binder, A Guide to Monte Carlo Simulations in Statistical Physics (Cambridge University Press, Cambridge, 2000).

19 G. Kresse and J. Hafner, Phys. Rev. B 47, 558 (1993).

20 G. Kresse and J. Furthmüller, Comput. Mat. Sci. 6, 15 (1996).

21 G. Kresse and J. Furthmüller, Phys. Rev. B 54, 11169 (1996).

22 J. P. Perdew, J. A. Chevary, S. H. Vosko, K. A. Jackson, M. R. Pederson, D. J. Singh, and C. Fiolhais, Phys. Rev. B 46, 6671 (1992).

23 J. P. Perdew and Y. Wang, Phys. Rev. B 45, 13244 (1992).

24 D. Vanderbilt, Phys. Rev. B 41, 7892 (1990). 
${ }^{25}$ G. Kresse and J. Hafner, J. Phys.: Condens. Matter 6, 8245 (1994).

26 M. P. Allen and D. J. Tildesley, Computer Simulation of Liquids (Oxford University Press, Oxford, UK, 1987).

27 S. Nosé, J. Chem. Phys. 81, 511 (1984).

28 Y. Waseda, The Structure of Non-Crystalline Materials - Liquids and Amorphous Solids (McGraw-Hill Inc., New York, 1980).

${ }^{29}$ H. Olbrich, H. Ruppersberg, and S. Steeb, Z. Naturf. 38a,
1328 (1983).

${ }^{30}$ H. K. Lee and R. H. Swendsen, Phys. Rev. B 64, 214102 (2001).

31 S. Duane, A. D. Kennedy, B. J. Pendleton, and D. Roweth, Phys. Lett. B 195, 216 (1987).

32 D. D. Frantz, D. L. Freeman, and J. D. Doll, J. Chem. Phys. 93, 2769 (1990). 